\title{
La incorporación de la cláusula social y el funcionamiento del mercado. Dudas sobre su efectividad
}

\author{
Armando Alvares Garcia Júnior
}

\section{RESUMEN}

El presente artículo analiza el grado de eficiencia de la denominada «cláusula social» (cláusula de protección de los derechos humanos y laborales) incorporada en los acuerdos bilaterales de comercio concluidos entre la Unión Europea y los países en desarrollo, tanto bajo la perspectiva del funcionamiento del mercado (primera parte del artículo) como de su aceptabilidad en el ámbito latinoamericano y europeo (segunda parte).

Palabras clave: Unión Europea, países en desarrollo, América Latina, cláusula social, mercado.

\section{The incorporation of the social clause and the market performance. Doubts about its effectiveness}

\section{Abstract}

This article analyzes the efficiency of the so-called «social clause» (clause of protection of human and labor rights) incorporated into bilateral trade agreements concluded between the European Union and developing countries, both from the perspective of the market performance (first part of the article) and its acceptability in the Latin American and European context (second part).

Keywords: European Union, developing countries, Latin America, social clause, market.

\footnotetext{
* Profesor de Derecho Internacional Público, Sistemas Jurídicos no Occidentales, Derecho del Comercio Internacional, Estructura Económica Mundial, Negocios Internacionales, Comercio Exterior, Negociación y Marketing internacional y Dirección Estratégica y Política de Empresa - I en la Facultad de Ciencias Jurídicas, Sociales y Humanidades de la Universidad Internacional de la Rioja (UNIR), Logrońo/Madrid, Espańa. Investigador del Grupo de Investigación «Justicia, Derecho y Globalización« (Globalaw) en la misma institución. Internacionalista. PhD. Posee tres doctorados (áreas de concentración: a) Derecho Internacional Económico, b) Derecho Empresarial Internacional, c) Derechos Humanos y Comercio Internacional) y diversos másteres y cursos de especialización (universidades de Harvard, Lund, Leiden, Ginebra, California, Pensilvania, Nuevo México, HEC París, Ínsper, IESE, ESADE etc.). Expresidente de la Asociación Brasileńa de Abogados para el Mercosur y exdirector de la Facultad de Derecho de la Universidad Bandeirantes de São Paulo, Brasil. Ha publicado 45 libros por editoriales de prestigio (principalmente en Brasil y España) y numerosos artículos en revistas científicas. Doctorando en Ciencias Políticas (Universidad Complutense de Madrid) y doctorando en Relaciones Internacionales Iberoamericanas (Universidad Rey Juan Carlos), ambas en Madrid, Espańa. Correo electrónico: armando.alvares@unir.net
} 


\section{La cláusula social y el libre mercado}

La cláusula social (cláusula de protección de los derechos humanos y laborales) incorporada en los acuerdos bilaterales de comercio Unión Europea (UE) - Países en Desarrollo (PED) no es la panacea. Al contrario, su eficiencia es mucho más reducida de lo que oficialmente reconoce la Comisión Europea.

El problema de la interconexión entre los derechos humanos y laborales (DD.HH.L) y el comercio internacional es de naturaleza esencialmente estructural. La Organización Mundial del Comercio (OMC) es parte integrante de esa gran estructura institucional de gobernanza global surgida con los acuerdos de Bretton Woods, sosteniendo y fomentando ciertas características que convierten esos derechos en algo verdaderamente escurridizo en la práctica de las relaciones comerciales internacionales ${ }^{1}$.

Naturalmente, existen defensores y detractores del libre comercio (todo depende de los beneficios potenciales que se considera posible obtener). Argumentos a su favor pueden ser encontrados, por ejemplo, en las obras de Bhagwati (2007), Grossman y Helpman (2015) o Harrison (2016). Sin embargo, el hecho de que el mundo esté globalizado, de que los miembros de la sociedad internacional estén hiperconectados y que exista una enorme interdependencia económica (la interconexión deriva, esencialmente, del comercio y de las finanzas ${ }^{2}$ ) no significa que los resultados sean beneficiosos para todos en el mismo grado (Dürrschmidt, 2015; Jensen, Quinn y Weymouth, 2016). De hecho, relativamente al comercio, cabe comentar que el nivel de renta influye en el modelo de producción y este en el volumen de exportaciones e importaciones, lo que tiende a afectar la actividad y el empleo en el resto de países. Además, algunas teorías defienden la existencia de una zona periférica (constituida por PED) dependiente de un centro (constituido por PD), cuya relación de dominancia (del centro con relación a la periferia) perpetuaría un desarrollo desigual y la consolidación de importantes asimetrías en las relaciones internacionales.

\footnotetext{
1 El libre comercio, una de las principales banderas erigidas después de la Segunda Guerra Mundial, es proclamado por los miembros de la OMC (actualmente 162) en sus distintas rondas de negociación (aunque la accidentada Ronda Doha haya puesto de manifiesto la relevancia y los conflictos de intereses existentes entre los países desarrollados/PD y los PED). Este objetivo también es perseguido por los diversos acuerdos internacionales que visan el establecimiento de espacios económicamente ampliados. No obstante, el multilateralismo en materia de comercio ha sido, de cierto modo, reemplazado por los numerosos acuerdos bilaterales preferenciales impulsados por las economías avanzadas al margen de la OMC. Además, muchos PED han optado por esta vía ante la dificultad de lograr un acuerdo general ambicioso. La Ronda de Doha, en su reunión ministerial de Nairobi (diciembre 2015) se considera prácticamente "muerta» en términos de una gran idea global, aunque temas como el acuerdo de Bali sobre facilitación de trámites comerciales, logrado en 2013, cuente ya con 65 ratificaciones (a fecha de 19.01.2016).

2 A sabiendas de que existen enormes interdependencias, sería conveniente la coordinación de políticas económicas entre los países para que todos obtengan un beneficio mutuo. Sin embargo, en tiempos de crisis económica los países se sienten tentados a utilizar esas interdependencias en beneficio propio.
} 
Para efectos de consagración de los DD.HH., objetivo asociado a la cláusula social, importa tener en mente la dificultad de compatibilizarlos con el modelo de asignación de recursos habitualmente adoptado por las empresas actuantes en el libre mercado, algo estimulado por el comercio internacional, la OMC, la UE y América Latina (AL). El escenario donde se desarrollan los intercambios mercantiles internacionales desvela un delicado campo por donde se mueven los DD.HH. en el comercio. Para mejor comprenderlo, es conveniente recordar que la actividad económica está directamente relacionada con la producción, el consumo y los intercambios comerciales (este último sufre, además, la influencia de diversos otros factores como los tipos de cambio entre divisas, tradicionalmente usados para estimular las exportaciones e incrementar la competitividad del país en el mercado mundial). En el seno de la UE, esas actividades deben tomar en cuenta los DD.HH. Lo mismo ocurre —o debería ocurrir- bajo la perspectiva de la UE, con los intercambios comerciales realizados con terceros países (desarrollados y en desarrollo). De hecho, la política exterior de la UE consagra como valores fundamentales los DD.HH., la democracia y el estado de derecho.

En la práctica, la dificultad está en que las relaciones comerciales UE-AL se desarrollan en un marco de libre mercado, en que la riqueza generada con el comercio no está condicionada estrictamente a los recursos naturales existentes, sino a la asignación apropiada y eficaz de los recursos económicos, humanos y materiales existentes (Hindriks y Myles, 2006; Sexton, 2015; McCluskey, Pasquale y Taub, 2016). Bajo esta óptica, la protección y el fomento de los DD.HH. (incluyendo los derechos laborales: DD.HH.L) son muchas veces relegados a un plano secundario en las decisiones relativas a la asignación de recursos. En efecto, las empresas de varios países, con el propósito de maximizar sus beneficios, tienden a vulnerar los DD.HH.L y a contar, algunas veces, con la condescendencia de ciertos gobiernos. Por ejemplo, la mano de obra barata (y frecuentemente sobreexplotada) configura, de acuerdo con Freeman y Nickell (1988), Mahmud (2015), Meyer y Peng (2016) y Rodgers (2016), uno de los más importantes factores de la competencia empresarial («liderazgo en costes») ${ }^{3}$. En el libre mercado, algo defendido por UE, AL y OMC, los agentes privados adoptan decisiones que objetivan maximizar sus beneficios económicos y reducir simultáneamente la correspondiente asignación de recursos. En otras palabras, en las economías de mercado, las decisiones económicas (lo que incluye la asignación de recursos) están basadas en decisiones privadas ${ }^{4}$ que se mueven esencialmente,

\footnotetext{
3 Algunos PED producen con bajos costes laborales y el precio competitivo de sus productos afecta a los puestos de trabajo en industrias de otros países.

4 Existen aproximadamente 104.000 multinacionales en el mundo, que controlan alrededor de 800.000 subsidios, $71 \%$ de ellas originarias de países desarrollados. Las 500 mayores empresas contabilizan el $25 \%$ de todo el producto
} 
según Arye Hillman (2009), en función de los beneficios económicos y de las informaciones pertinentes disponibles (en el mismo sentido, Markus y Jacobson, 2015; Rangan, 2015; Culpepper, 2016; Rothlin y McCann, 2016 y Deeg y Jackson, 2016).

La transposición de la cláusula social incorporada en un acuerdo comercial internacional en la práctica empresarial enfrenta ese problema. Este es el contexto liberalizador en que se insieren las empresas de la UE y de AL y que viene siendo sostenida en el ámbito multilateral del comercio desde los acuerdos de Bretton Woods y la creación del GATT, predecesor de la OMC. Por eso, bajo la perspectiva del mercado, la cláusula social es ineficiente. Las decisiones estratégicas empresariales buscan la maximización de los beneficios, lo que significa que, si es necesario o simplemente conveniente, dependiendo del sector y del tipo de mano de obra (especialmente la poco calificada), no pondrán reparos en precarizar las condiciones laborales.

Se podría pensar que el Estado debería luchar rotundamente para evitar situaciones como esas. De hecho, la incorporación de la cláusula social en los acuerdos comerciales bilaterales concluidos por la UE con países o subregiones en desarrollo (v.g. Centroamérica), establece la ratificación y aplicación efectiva de determinados instrumentos internacionales (considerados como el mínimo aceptable por la UE en términos de estándares de protección de los DD.HH.L) como una condicionante para acceder al mercado único europeo con rebajas arancelarias.

Aquí también, en la práctica, la cláusula social no es tan eficiente como se imagina. Ni siempre existe un interés estatal real para evitar esta situación. Esto ocurre porque casi todos los gobiernos basan el sistema de desarrollo del país en la exportación y en la competitividad de sus empresas, mirando algunas veces hacia otro lado en temas de violaciones de los DD.HH.L perpetradas por los agentes privados 5 , algo contemplado, a su manera, desde hace varios ańos, por los investigadores Collingsworth, Goold y Harvey (1994) y De Wet (1995) y, más recientemente, por Chen (2015), Irwin (2015) y Hansson (2016) entre otros. Bajo el enfoque del mercado, la razón es sencilla: las exportaciones son beneficiosas para el equilibrio de la balanza comercial y de pagos. Con suerte, la cláusula social tendrá un efecto positivo en la promoción de los DD.HH.L en los países signatarios económicamente más débiles,

\footnotetext{
(bienes y servicios) en el mundo y el 50\% de todo el comercio mundial. La mayor parte de la tecnología en el mundo - y sus patentes - también están en sus manos. El $80 \%$ de los pagos por royalties y tasas involucran a esas grandes empresas. Fuente: Wharton Business School, Universidad de Pensilvania. Curso sobre Análisis de las tendencias financieras y sociales a nivel global, impartido por Mauro Guillén, de 05 de mayo a 02 de julio de 2014. Apuntes personales.

5 La violación de derechos civiles, políticos, económicos, sociales y culturales es una realidad palpable en muchos países que se insiere dentro de esa lógica. Empresas y gobiernos son actores partícipes en este campo.
} 
puesto que el Estado, antes de firmar y ratificar un acuerdo comercial con la UE y vincularse jurídicamente a la cláusula social (normalmente considerada una cláusula fundamental en los acuerdos, y cuya violación puede generar —en función de la aplicación de la Convención de Viena sobre Derecho de los Tratados- sanciones de diferentes tipos), pondera si merece la pena exportar sus productos al mercado europeo. La respuesta, naturalmente, suele ser afirmativa.

Teóricamente, la situación de vulneración de los DD.HH.L debería reducirse drásticamente cuando el país ratifica los instrumentos internacionales pertinentes (señalados, por ejemplo, en el anexo VIII del Reglamento no 978/2012 del Parlamento Europeo y del Consejo, de 25 de octubre de 2012). En la práctica, sin embargo, cuando el compromiso político del Estado se transfiere (sin excluir la responsabilidad estatal) a la actividad privada, suele ser ignorado o, algunas veces, se convierte en algo contraproducente (v.g., algunas empresas recortan la plantilla de empleados por reducción de la competitividad en la etapa de producción). Cuando se procede al análisis económico del derecho se observa que la cláusula social podría estar, incluso, indirectamente asociada al incremento de despidos en un país (Kaplow y Shavell, 2002, Becker, 2013, Richter, 2015, Gabaix, 2016, Ahn, 2016). Esto no es ninguna novedad puesto que en los PED, tradicionalmente, cuanto más normas laborales favorables al trabajador se editan, menor la contratación formal en el mercado de trabajo.

Así, por un lado, el Estado desea y necesita fomentar la exportación de bienes y servicios producidos por sus empresas y, por otro, teme violar el acuerdo concluido con la UE (puesto que está jurídicamente vinculado a su cláusula social), porque de esa violación derivarían responsabilidades internacionales. A nivel bilateral, la consecuencia principal sería la restricción comercial mediante la retirada de concesiones comerciales favorables a esos PED. Como se sabe, desde el inicio de los años setenta (1971), la UE busca estimular a los PED para que ratifiquen tratados de protección de los DD.HH. y determinados convenios de la Organización Internacional del Trabajo (OIT). La cláusula social incorporada en los acuerdos comerciales bilaterales concluidos por la UE con esos países cumple un rol condicionante en lo que concierne al acceso facilitado de sus productos al mercado interior europeo (para recibir la rebaja arancelaria debe el PED incorporar en su ordenamiento jurídico esos instrumentos internacionales asociados a la cláusula social).

No obstante, la realidad empresarial en Latinoamérica, especialmente en las zonas más pobres, aún revela un cuadro de precariedad elevada. Además, en un marco de libre competencia, otro icono neoliberal, de poco serviría exportar si no se logran precios competitivos que permitan abrir un hueco en el mercado exterior y, gradualmente, 
ampliar su cuota de participación ${ }^{6}$. Por otro lado, la mayoría de los consumidores raramente piensa en adquirir productos más caros, solo porque su producción se ha realizado con el escrupuloso respeto de las normas laborales y la protección de los derechos civiles, políticos, económicos, sociales y culturales de las personas de este o de aquel país. Simplemente compran lo que es económicamente accesible para sus bolsillos. Esto, en microeconomía, corresponde al principio de la maximización de resultados en función de la restricción presupuestaria del consumidor, considerado un agente racional.

Ahora, ¿̇será que los Estados son indiferentes con relación al modo como los DD.HH. son encarados y asumidos por sus agentes privados en el libre mercado? Teóricamente no. Por coherencia, esta idea sería extraña, puesto que hasta la asignación de recursos por el Estado (recursos públicos), realizados mediante la edición de normas jurídicas, debe evitar la vulneración de los DD.HH. ${ }^{7}$. Desde luego, el rol de los agentes privados en el tema de los DD.HH.L es relevante para la UE, como se puede observar, por ejemplo, con la Resolución del Parlamento Europeo, de 26 de marzo de 2009, sobre la responsabilidad social de las empresas de subcontratación en las cadenas de producción (2008/2249(INI)), la Resolución de 25 de noviembre de 2010, sobre la responsabilidad social de las empresas en los acuerdos de comercio internacional (2009/2201(INI)) o la de 8 de junio de 2011, sobre la dimensión exterior de la política social, la promoción de las normas laborales y sociales y la responsabilidad social de las empresas europeas (2010/2205(INI)). Existen varios otros instrumentos jurídicos que versan el tema, como el Dictamen del Comité Económico y Social Europeo sobre "Transformaciones industriales, desarrollo territorial y responsabilidad de las empresas» o la Resolución del Consejo de 6 de febrero de 2003 relativa a la responsabilidad social de las empresas. Para predicar con el ejemplo se podría mencionar aun el Dictamen del Comité Económico y Social Europeo sobre la «Comunicación de la Comisión al Parlamento Europeo,

\footnotetext{
6 El Reglamento (CE) no 1225/2009 del Consejo de 30 de noviembre, relativo a la defensa contra las importaciones que sean objeto de dumping por parte de países no miembros de la Comunidad Europea (Diario Oficial de la Unión Europea de 22.12.2009 (L 343/51) es un ejemplo de esta preocupación, respaldada por los tribunales (v.g. la Sentencia 62012TJ0006, del Tribunal General (Sala Cuarta) de 6 de septiembre de 2013 (Godrej Industries Ltd y VVF Ltd contra Consejo de la Unión Europea, 06.09.2013 (ECLI:EU:T:2013:408). Sobre productos específicos, se puede mencionar el Reglamento de Ejecución (UE) 2015/501 de la Comisión de 24 de marzo de 2015 que impone un derecho antidumping provisional sobre las importaciones de productos planos de acero inoxidable laminados en frío originarios de la República Popular China y Taiwán (Diario Oficial de la Unión Europea de 25 de marzo de 2015 (L 79/23)).

7 Todo el tema es tomado muy en serio por la Unión Europea como revela, por ejemplo, la Resolución B4-0731, 0733, 0742, 0760 y 0778/98 sobre la violación de los derechos humanos en Myanmar,el Dictamen del Comité Económico y Social Europeo sobre la «Comunicación de la Comisión al Parlamento Europeo, al Consejo, al Comité Económico y Social Europeo y al Comité de las Regiones sobre la estrategia renovada de la UE para 2011-2014 sobre la responsabilidad social de las empresas».
} 
al Consejo y al Comité Económico y Social Europeo - Poner en práctica la asociación para el crecimiento y el empleo: hacer de Europa un polo de excelencia de la responsabilidad social de las empresas». Estas disposiciones legales siguen las teorías éticas y morales en los negocios, señalados por Mayorga y González (2001), Boesso, Favotto y Michelon (2015) o Corkery, Mikalsen y Allan (2016) y muy conocidas en el ámbito de la Administración y Dirección de Empresas. En resumen: la eficiencia de la cláusula social es relativa, bajo la perspectiva del funcionamiento real del mercado.

\section{Derechos Humanos y Comercio Internacional: Aceptabilidad de la cláusula social en América Latina}

Históricamente los Estados latinoamericanos (así como una parcela significativa de sus respectivas sociedades ${ }^{8}$ ) se posicionan contrariamente a la inclusión de los DD.HH.L en el ámbito de los acuerdos comerciales internacionales, tanto bilaterales como multilaterales. En el marco del sistema multilateral de comercio, el repudio latinoamericano ha quedado marcado de forma muy intensa durante las negociaciones de la Ronda Uruguay del GATT, a tal punto que el gobierno estadounidense llegó a acusar a esos países de practicar el denominado dumping social al obtener ventajas comerciales sobre los países que adoptan estándares laborales más elevados (Jinji, 2005; Arnholtz y Eldring, 2015; Xu, 2016), lo que configuraría una especie de «competencia desleal» (Cordella y Grilo, 2001; Verschueren, 2015; Ewing, 2016; Savona y Oldani, 2016).

En el ámbito de la UE el tema se discute tanto a nivel de intrazona como en relación con terceros países (v.g., Pregunta escrita P-8845/10 Emilie Turunen (Verts/ALE) a la Comisión. Dumping social; Pregunta escrita E-8805/10 Oriol Junqueras Vies (Verts/ ALE) a la Comisión. Denuncia de dumping económico, social y sanitario de la industria conservera de Galicia contra la Comisión Europea; Pregunta escrita E-7704/10 Othmar Karas (PPE) a la Comisión. Dumping salarial en Alemania: empresas de sacrificio y despiece). En la Ronda de Singapur, en 1996, ya estando constituida y actuante la OMC, Estados Unidos de América (EE.UU.) y Noruega intentaron incluir nuevamente el tema de la cláusula social en la pauta de las negociaciones multilaterales, pero los PED lograron bloquear la iniciativa, aludiendo a la competencia de la OIT, explícitamente señalada, un año antes, por su primer presidente,

\footnotetext{
8 La cláusula social incorporada en un acuerdo comercial no encuentra necesariamente respuesta positiva por parte de los agentes privados, lo que incluye tanto a las empresas como a los propios trabajadores, que temen perder sus puestos de trabajo.
} 
el napolitano Renato Ruggiero9. En esta Conferencia se proclamó la siguiente Declaración Ministerial:

We renew our commitment to the observance of internationally recognized core labour standards. The International Labour Organization (ILO) is the competent body to set and deal with these standards, and we affirm our support for its work in promoting them. We believe that economic growth and development fostered by increased trade and further trade liberalization contribute to the promotion of these standards. We reject the use of labour standards for protectionist purposes, and agree that the comparative advantage of countries, particularly low-wage developing countries, must in no way be put into question. In this regard, we note that the WTO and ILO secretariats will continue their existing collaboration ${ }^{10}$.

De este modo, en el marco del sistema multilateral de comercio, la cláusula social se convertiría en un punto de discordia entre los PD y los PED. Los primeros acusaban a los segundos de competencia desleal y los segundos acusaban a los primeros, de acuerdo con Elmslie y Milberg (1996), Christodoulidis (2016) y Niranjan, (2016) de ocultar un verdadero proteccionismo comercial por detrás de su discurso sobre DD.HH. De un modo general, los PED repudiaban la vinculación de la protección de los DD.HH.L a los acuerdos comerciales al considerarla una estrategia articulada por los PD para neutralizar la mayor ventaja comparativa que poseían: su mano de obra abundante y barata (Appelbaum y Christerson, 1997 y Roozendaal, 2002; Zahn y Mangan, 2015; Wilcock y Scholz, 2016).

Si se analiza la cláusula social bajo la perspectiva de su plena aceptabilidad por todos los pueblos (puesto que plasman valores supuestamente universales), se verá, una vez más, que en la práctica eso no ocurre. La dimensión social del comercio era, bajo la perspectiva de los PED, una estrategia proteccionista, comprendida y explorada en AL por algunos segmentos de la sociedad y de la clase política, como la expresión de la rivalidad entre los países del Norte y del Sur, posicionamiento ya destacado en los ańos setenta del siglo pasado por Diaz-Alejandro (1975). En el ámbito de la UE, como señala Gough (1999 y 2016), se llegó a abordar el tema bajo la perspectiva de la cohesión social. En términos normativos, se podría proporcionar como ejemplos el Dictamen del Comité Económico y Social Europeo sobre el tema «La dimensión social de la globalización - la contribución de la política comunitaria para que los beneficios se extiendan a todos», de 09.03.2005 o, más recientemente, la Comunicación de la Comisión al Parlamento Europeo, al Consejo, al Comité Económico

\footnotetext{
9 No obstante, hay que considerar que la OIT no posee poderes sancionadores, aunque exista reclamación de otro país-miembro (non-observance complaints).

${ }_{10}$ Organización Mundial del Comercio. Doha WTO Ministerial 2001: Briefing Notes. Trade and Labour Standards. A difficult issue for many WTO member governments. Recuperado de http://www.wto.org/english/thewto_e/ minist_e/min01_e/brief_e/brief16_e.htm
} 
y Social Europeo y al Comité de las Regiones sobre «La dimensión social de la globalización: la contribución de la política comunitaria para que los beneficios se extiendan a todos", de 18.05.2012; o el Dictamen del Comité Económico y Social Europeo sobre la Comunicación de la Comisión al Parlamento Europeo, al Consejo y al Comité Económico y Social Europeo sobre «Comercio, crecimiento y desarrollo: una política de comercio e inversión a medida para los países más necesitados», de 18.09.2012.

Es importante enfatizar que en el ámbito del sistema multilateral de comercio, por iniciativa del propio Sr. Ruggiero, la apreciación de los temas laborales sería transferida al marco competencial de la OIT que, en aquel momento, estaba debatiendo una propuesta de trabajo conjunto con la OMC (Leary, 1997, Krämer, 2016). No obstante, fuera del marco multilateral, sí que ha sido posible incorporar algunos convenios básicos de la OIT en instrumentos jurídicos internacionales. De hecho, la UE en sus relaciones comerciales con terceros países incluye varios de ellos (v.g., libertad sindical y derecho de asociación), comprendidos en el ámbito de la cláusula social.

Naturalmente, no era sencillo políticamente afirmar que la cláusula social correspondía a una política premeditada y deliberada para marginar a los PED y cristalizar las asimetrías observables entre los países en términos de desarrollo económico. Al contrario, como recuerdan Yusuf (1980), Hoekman y Mavroidis (2015) y McDonald (2016), desde el final de los años setenta, mediante la cláusula de habilitación (Ronda Tokio), se habían tomado iniciativas para mejorar la inserción comercial de esos países, visando su desarrollo socioeconómico ${ }^{11}$.

Por parte del bloque europeo, en el ámbito bilateral, tampoco era posible afirmar rotundamente su propósito de marginar a los PED. Su primera iniciativa de instituir el SPG (1971), años antes de que el GATT lograse un marco normativo multilateral $(1979)^{12}$, parece apuntar para la conformación de una imagen alternativa y diferenciada de aquella percibida en AL en relación con los EE.UU. La imagen de socio fiable,

\footnotetext{
11 Esta posición incluso se ha plasmado en el Reglamento no 978/2012 del parlamento Europeo y del consejo, de 25 de octubre, en su «considerando» $n^{\circ} 4$ : «La política comercial de la Unión debe ser coherente con los objetivos de la política de la Unión en el ámbito de la cooperación para el desarrollo y consolidar dichos objetivos, fijados en el artículo 208 del Tratado de Funcionamiento de la Unión Europea (TFUE), en particular la erradicación de la pobreza y la promoción del desarrollo sostenible y la gobernanza en los países en vías de desarrollo. Asimismo, debe cumplir los requisitos de la Organización Mundial del Comercio (OMC), especialmente la Decisión sobre trato especial y diferenciado, reciprocidad y mayor participación de los países en desarrollo (la «Cláusula de Habilitación»), adoptada en virtud del Acuerdo General sobre Aranceles Aduaneros y Comercio (GATT) en 1979, según la cual los miembros de la OMC pueden conceder un trato diferenciado y más favorable a los países en vías de desarrollo».

12 El Reglamento (UE) no 539/2014 del Parlamento Europeo y del Consejo, de 16 de abril de 2014, relativo a las importaciones de arroz originario de Bangladés y por el que se deroga el Reglamento (CEE) nº 3491/90 del Consejo alude explícitamente en su considerando $\mathrm{n}^{\circ} 8$ a la cláusula de habilitación.
} 
no imperialista, interesado en la promoción de los DD.HH, del estado de derecho, de la democracia, del desarrollo socioeconómico sería un logro a alcanzar, aunque nunca haya convencido plenamente a los socios más pobres de la OMC, como señalaron Kwa (1998), Porter (2015) y Siddiqui (2016). La verdad es que esa pretendida imagen del bloque europeo no era compatible del todo con la actuación de la UE en el marco de la OMC, puesto que la entidad había adoptado un modelo de negociación, en la práctica, en que los temas más relevantes y de interés para los PED (como la agricultura), eran discutidos casi que exclusivamente por los miembros desarrollados. Los PED recibían un texto prácticamente cerrado para que discutiesen (con poca margen de maniobrabilidad) los aspectos técnicos de menor importancia. En el marco bilateral la situación era similar. La propia Oxfan $(2002)^{13}$ ha publicado un informe ( $\left.n^{\circ} 22\right)$ que establecía en su página inicial:

Los dobles raseros en política comercial son una vergüenza. La UE está forzando a los países del Sur a abrir sus mercados a una velocidad suicida, al tiempo que mantiene sus propias barreras a las exportaciones de estos países, en especial a los productos agrarios y textiles. Pero la UE aún va más allá, invadiendo el mundo en desarrollo con sus excedentes agrarios fuertemente subvencionados a unos precios con los que los pequeños productores de estos países no pueden competir, destruyendo con ello su medio de vida.

Tomando en cuenta que: a) la Decisión de 1979 sobre un trato diferenciado más favorable, reciprocidad y mayor participación de los PED (cláusula de habilitación) ha sido plasmada en el artículo XXIV del General Agreement on Tariffs and Trade/GATT (como se desprende del Entendimiento sobre la Interpretación del Artículo XXIV del GATT 1994); b) la Resolución del Parlamento Europeo sobre las Zonas Regionales de Libre Comercio y la Estrategia Comercial de la UE (2002/2044(INI)) ${ }^{14}$ señala, en su letra «I», la necesidad de que las negociaciones sobre los acuerdos comerciales regionales esclarezcan las disposiciones sobre la flexibilidad existente en el marco de la OMC, por un lado, y la relación entre el artículo XXIV del GATT y la «cláusula de habilitación», por otro lado; c) en su letra «O» (de la Resolución del Parlamento Europeo sobre las Zonas Regionales de Libre Comercio y la Estrategia Comercial de la UE: 2002/2044(INI) exprese sus preocupaciones sobre el incremento de los intercambios comerciales en el ámbito Sur-Sur, entre países aun beneficiados por la cláusula de habilitación del GATT ${ }^{15}$ y d) tanto la OMC como la UE y AL proclaman tanto el libre comercio como la libre competencia (con todos los aspectos ya

\footnotetext{
13 Oxfam. Informe Oxfan no 22. (2002). La hipocresía de Europa. Recuperado de http://www.fongdcam.org/ manuales/comerciojusto/datos/docs/ARTICULOS\%20Y\%20DOCUMENTOS\%20DE\%20REFERENCIA\%20 def/CONCEPTO\%20CI/Como\%20funciona\%20el\%20sistema/11-Hipocresia_Europa_oxfam.pdf

14 Entre otros instrumentos.

15 Preocupación expresada, por ejemplo, en la Decisión de la Comisión de 2 de mayo de 2005 relativa a las medidas necesarias en relación con el obstáculo al comercio que constituyen las prácticas comerciales impuestas por Brasil al comercio de neumáticos recauchutados (considerando no 6). Diario Oficial de la Unión Europea de 21.05.2005 (L 128/71).
} 
mencionados sobre la maximización de beneficios económicos por las empresas, la connivencia de muchos gobiernos, los planes de desarrollo nacional basado en las exportaciones, el mejoramiento de la balanza comercial y de pagos etc.) se comprenderá que todo el escenario es complejo. Naturalmente, no hay como la cláusula social pueda ser muy eficiente en este entorno.

\section{Conclusiones}

La cláusula social incorporada en los acuerdos comerciales concluidos por la UE con los PED atiende a un discurso políticamente correcto. No obstante, enfrenta dificultades para lograr su principal objetivo — promover los DD.HH.L en esos países- porque, de un modo general, el mercado se basa en otros mecanismos y fundamentos. Por ejemplo, el libre comercio y la libre competencia, proclamados por la OMC, la UE y AL, están estrechamente asociados a la idea de asignación de recursos por parte de los agentes privados (lo que puede incluir bajos salarios, explotación de mano de obra, utilización de trabajo infantil, etc.), aspectos analizados en la primera parte del artículo. Además del enfoque en las empresas (y en menor escala en la connivencia de los Estados), también se apreció —en la segunda parte de este trabajo- el tema de la aceptabilidad de la inclusión de la cláusula social en esos acuerdos, siendo señalado tanto las posiciones generales de las regiones en desarrollo (en contra) como de las regiones desarrolladas (a favor).

\section{Bibliografía}

Ahn, Dukgeun (2016). The Legal and Economic Analysis of the WTO/FTA System. London, World Scientific Pub Co Inc. http://dx.doi.org/10.1142/9708

Appelbaum, Richard y Christerson Brad (1997). Cheap labor strategies and export-oriented industrialization: some lessons from the Los Angeles/East Asia apparel connection. International Journal of Urban and Regional Research, 21(2), 202-217.

Arnholtz, Jens y Line Eldring (2015). 4 Varying perceptions of social dumping in similar countries. Market Expansion and Social Dumping in Europe, 80, 21-31.

Arye, Hillman (2009). Public Finance and Public Policies. Responsibilities and Limitations of Government. Cambridge University Press.

Becker, Gary S. (2013). The economic approach to human behavior. University of Chicago Press.

Bhagwati, Jagdish (2007). In Defense of Globalization. Oxford: Oxford University Press.

Boesso, Giacomo, Francesco Favotto y Giovanna Michelon (2015). Stakeholder prioritization, strategic corporate social responsibility and company performance: Further evidence. Corporate Social Responsibility and Environmental Management, 22(6), 424-440. http://dx.doi.org/10.1002/csr.1356 
Chen, Haoqian (2015). Social Clause in Trade Agreements and China’s Experience. Beijing Law Review, 6(1), 1-13. http://dx.doi.org/10.4236/blr.2015.61001

Collingsworth, Terry, William Goold y Pharis Harvey (1994). Labor and free trade: time for a global new deal. Foreign Affairs, 73(1), 8-13. http://dx.doi.org/10.2307/20045886

Corkery, Jim, Mikalsen Maiken y Katie Allan (2016). Corporate social responsibility: The good corporation. Gold Coast. Centre for Commercial Law, Bond University.

Cordella, Tito e Isabel Grilo (2001). Social dumping and relocation: is there a case for imposing a social clause? Regional Science and Urban Economics, 31(6), 643-668. http:// dx.doi.org/10.1016/s0166-0462(01)00063-1

Christodoulidis, Emilios (2016). A Default Constitutionalism? A Disquieting Note on Europe's Many Constitutions. The Many Constitutions of Europe, 31-44.

Culpepper, Pepper D. (2016). Capitalism, Institutions, and Power in the Study of Business. The Oxford Handbook of Historical Institutionalism.

Deeg, Richard y Gregory Jackson (2016). Changing Models of Capitalism in Europe and the US. Routledge.

De Wet, Erika (1995). Labor standards in the globalized economy: the inclusión of a social clause in the General Agreement on Tariff and Trade/World Trade Organization. Human Rights Quartely, 443-462.

Díaz-Alejandro, Carlos F. (1975). North-south relations: The economic component. International Organization, 29(01), 213-241. http://dx.doi.org/10.1017/ S002081830001794X

Dürrschmidt, Jörg (2015). Globalization and Existential Inequality: The Precariousness of Belonging. Understanding the Dynamics of Global Inequality. Berlin/Heidelberg. Springer. http://dx.doi.org/10.1007/978-3-662-44766-6_14

Elmslie, Bruce y William MILBERG (1996). Free trade and social dumping: lessons from the regulation of US interstate commerce. Challenge 72, 46-52.

Ewing, Keith D. (2016). Transnational Labour Law. King's Law Journal, 27(1), 132-135. http://dx.doi.org/10.1080/09615768.2016.1163907

Freeman, Richard y Stephen Nickell (1988 Labour market institutions and economic performance. Economic Policy, 136, 64-80. http://dx.doi.org/10.2307/1344504

Gabaix, Xavier (2016). Power Laws in Economics: An Introduction. The Journal of Economic Perspectives, 30(1), 185-205. http://dx.doi.org/10.1257/jep.30.1.185

Gough, Ian (1999). Capitalism and social cohesion: essays on exclusion and integration. Macmillan.

Gough, Ian (2016). Global capital, human needs and social policies. Springer.

Grossman, Gene M. y Elhanan Helpman (2015). Globalization and Growth. The American Economic Review, 105(5), 100-104. http://dx.doi.org/10.1257/aer.p20151068

Hansson, Göte (2016). Trade and Labour Standards. The Assignee Research Companion to International Trade Policy, 279, 61-82.

Harrison, Graham (2016). Global Encounters: International Political Economy, Development and Globalization. Springer. 
Hindriks, Jean y Gareth Myles (2006). Intermediate public economics. Cambridge: MIT Press. Hoekman, Bernard M. y Petros C. Mavroidis (2015). World Trade Organization (WTO): Law, Economics, and Politics. Routledge.

Irwin, Douglas A. (2015). Free trade under fire. Princeton University Press.

Jensen, J. Bradford, Dennis P. Quinn y Stephen Weymouth (2016). Winners and Losers in International Trade: The Effects on US Presidential Voting ( $\mathrm{n}^{\circ}$ 21899). National Bureau of Economic Research.

Jinji, Naoto (2005). Social Dumping and International Trade. ETSG Working Paper.

Kaplow, Louis y Steven Shavell (2002). Economic analysis of law. Handbook of Public Economics, 3, 1661-1784.

Krämer, Rike (2016). Balancing Human Rights, Environmental Protection and International Trade, Lessons from the EU Experience. Journal of International Economic Law, 19(1), 283-287. http://dx.doi.org/10.1093/jiel/jgw003

Kwa, Aileen (1998). WTO and Developing Countries, Foreign Policy in Focus, 3, 37, noviembre.

Leary, Virginia A. (1997). WTO and the Social Clause: Post-Singapore, The European Journal of International Law, 8, 118-122. http://dx.doi.org/10.1093/oxfordjournals.ejil.a015539

McDonald, Brian (2016). The world trading system: the Uruguay Round and beyond. Springer.

Mahmud, Tayyab (2015). Precarious Existence and Capitalism: A Permanent State of Exception. Southwestern University Law Review (15), 12-19.

Markus, M. Lynne y Dax D. Jacobson (2015). The governance of business processes. In Handbook on Business Process Management 2 (311-332). Berlin/Heidelberg. Springer

Mayorga Ospina, Pilar y Jaime Volmar González (2001). Responsabilidad social de la empresa. Elementos teóricos y experiencias. Fundación Corona.

Mccluskey, Martha T., Frank A. Pasquale y Jennifer Taub (2016). Law and Economics: Contemporary Approaches. Yale Law \& Policy Review, Forthcoming.

Meyer, Klaus y Mike Peng (2016). International Business. Cengage Learning.

Niranjan, Sunil Kumar (2016). WTO and Labour Issues: a challenge. Humanities \& Social Sciences Reviews, 4(1), 49-52.

OXFAM (2002). Informe Oxfan no 22. La hipocresía de Europa. Recuperado de http:// www.fongdcam.org/manuales/comerciojusto/datos/docs/ARTICULOS\%20Y\%20 DOCUMENTOS\%20DE\%20REFERENCIA\%20def/CONCEPTO\%20CI/ Como\%20funciona\%20el\%20sistema/11-Hipocresia_Europa_oxfam.pdf

Porter, Roger (2015). The World Trade Organization at Twenty. The Brown Journal of World Affairs, 21(2), 104-117.

Rangan, Subramanian (2015). Performance and Progress: Essays on Capitalism, Business, and Society. OUP Oxford.

Richter, Rudolf (2015). The Role of Law in the New Institutional Economics in Comparison with the Economic Analysis of Law. In Essays on New Institutional Economics (77-96). Springer International Publishing. http://dx.doi.org/10.1007/978-3-319-14154-1_4 
Rodgers, Lisa (2016). Labour Law, Vulnerability and the Regulation of Precarious Work. Edward Elgar Publishing. http://dx.doi.org/10.4337/9781784715755

Roozendaal, Gerda van (2002). Trade Unions and Global Governance: The Debate on a Social Clause. Londres: Routledge.

Rothlin, Stephan y Dennis Mccann (2016). Moral Decision-Making in Business. In International Business Ethics (65-89). Berlin-Heidelberg. Springer. http://dx.doi. org/10.1007/978-3-662-47434-1_4

Savona, Paolo y Chiara Oldani (2016). Globalisation, Growth, and Health: The Private Sector Perspective. Sustaining Global Growth and Development: G7 and IMF Governance, 99-121.

Sexton, Robert (2015). Exploring economics. Cengage Learning.

Siddiqui, Kalim (2016). The Political Economy of Free Trade, WTO and the Developing Countries. Turkish economic review, 3(1), 103-121.

Verschueren, Herwig (2015). The European internal market and the competition between workers. European Labour Law Journal, 6(2), 128-151.

Wilcock, Neil y Corina Scholz (2016). A Convoy Model of Globalisation. In Hartmut Elsenhans and a Critique of Capitalism (57-74). Palgrave Macmillan UK. http://dx.doi.org/ 10.1007/978-1-137-56464-1_5

$\mathrm{Xu}$, Yu-hong (2016). Reflection on Labor Standard's Linkage to International Trade. International Journal of Economics and Finance, 8(3), 165-173. http://dx.doi.org/10.5539/ ijef.v8n3p165

Yusuf, Abdulqawi A. (1980). Differential and More Favourable Treatment: The GATT Enabling Clause. Journal of World Trade, 14(6), 488-507.

Zahn, Rebecca y David Mangan (2015).Labour Standards and Sustainable Development: Unpicking the EU's Approach. International Journal of Comparative Labour Law and Industrial Relations, 31(3), 233-241.

Fecha de recepción: 19 de enero de 2016 Fecha de aprobación: 28 de abril de 2016 